\title{
La 3D numérique au cinéma
}

\author{
Laurent BLONDÉ \\ Technicolor - Research \& Innovation - Rennes \\ laurent.blonde@rechnicolor.com
}

La perception de la profondeur est une composante fondamentale de notre rapport au monde, tout du moins à l'espace qui nous entoure. Se déplacer, anticiper un mouvement ou une trajectoire, comprendre une scène complexe ou encore saisir un objet, voici autant de situations qui demandent une estimation, même approximative, de la profondeur. Aisée pour certains, plus ardue pour d'autres, la perception de la profondeur combine divers indices de perception, visuels - monoculaires ou binoculaires, et oculomoteurs (figure 1). De tous temps, les artistes ont essayé de rendre cette notion de profondeur en utilisant un certain nombre $d^{\prime}$ artifices : perspective, flou de distance, taille relative, taille et forme familières des objets, dégradé de textures, diffusion atmosphérique, autant d'éléments qui permettent de hiérarchiser les distances et de comprendre une scène dans sa complexité spatiale. Aujourd'hui, l'évolution des techniques 3D permet, grâce à plusieurs types de systèmes, de restituer cette profondeur de manière dynamique.

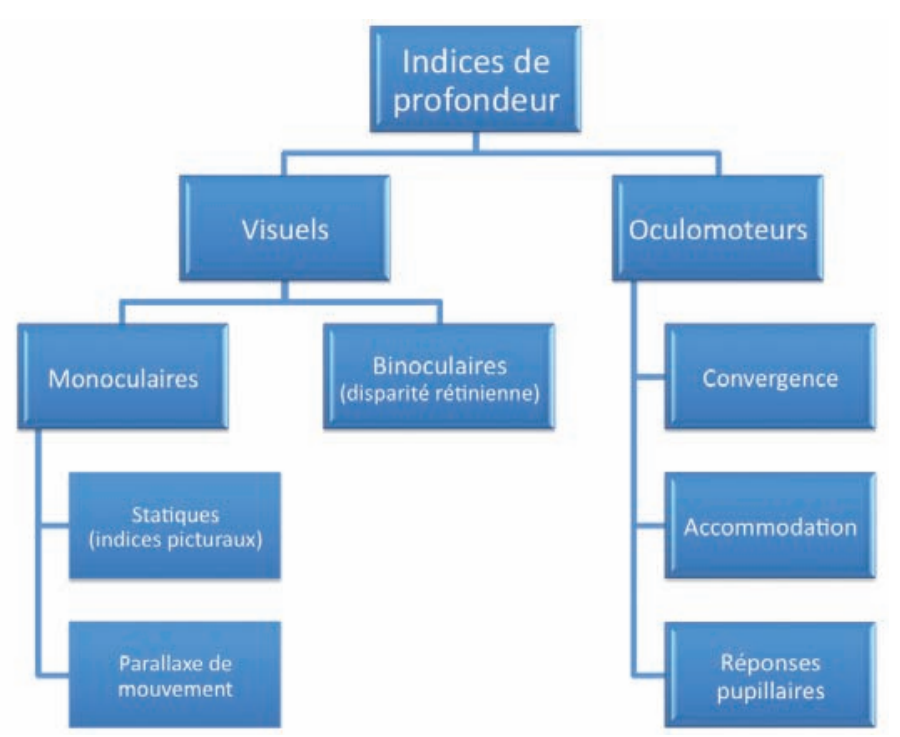

Figure 1. De nombreux indices permettent de percevoir la profondeur d'une scène.

\section{Perception de la profondeur}

\section{Les indices et la vision monoculaires}

Les artistes ont très tôt représenté une information de la profondeur dans leurs œuvres, et, partant de l'indice d'occlusion qui indique simplement qu'un objet est devant l'autre, on retrouve tous les indices monoculaires dans la peinture réaliste à travers les siècles, ou aujourd'hui dans la photographie (figure 2). Correspondant à une perception naturelle de l'espace, ce
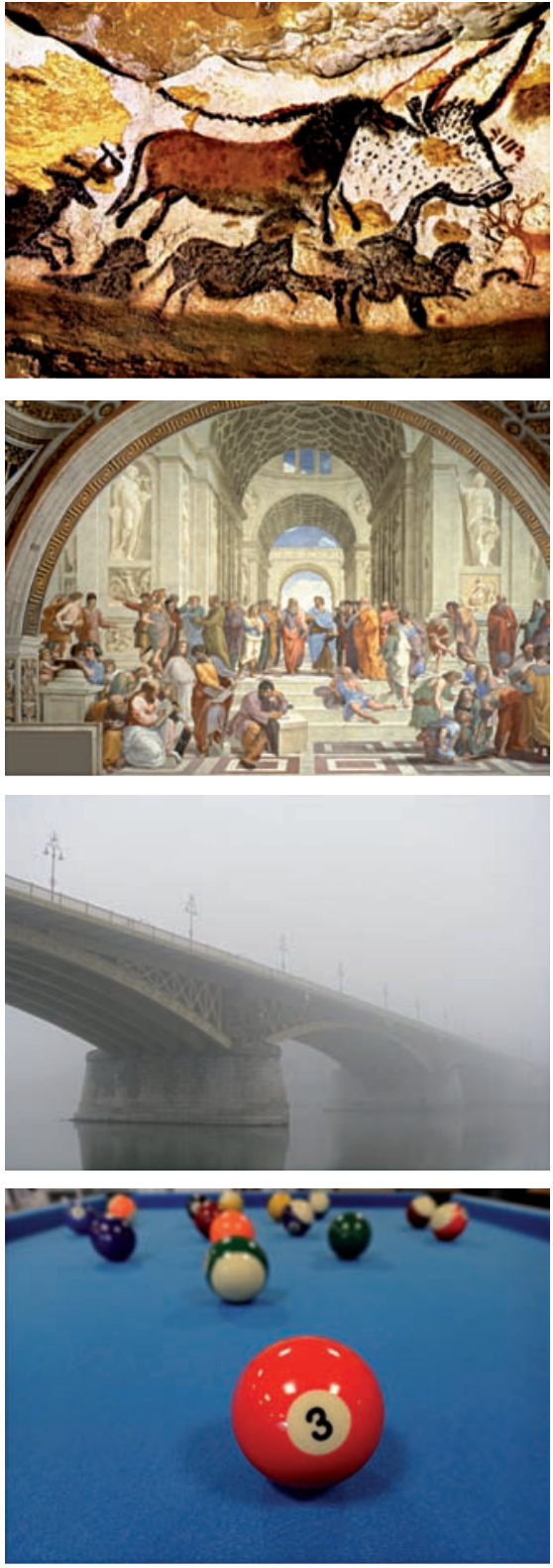

Figure 2. De tous temps, les hommes ont essayé de restituer la perception de la profondeur.

sont là des codes de perception classiques qui, lorsqu'ils ne sont pas congruents, interpellent le spectateur. Par exemple un dégradé de flou en avant plan et en arrière-plan obtenu avec un appareil à basculement d'objectif (tilt-shift) ou par 


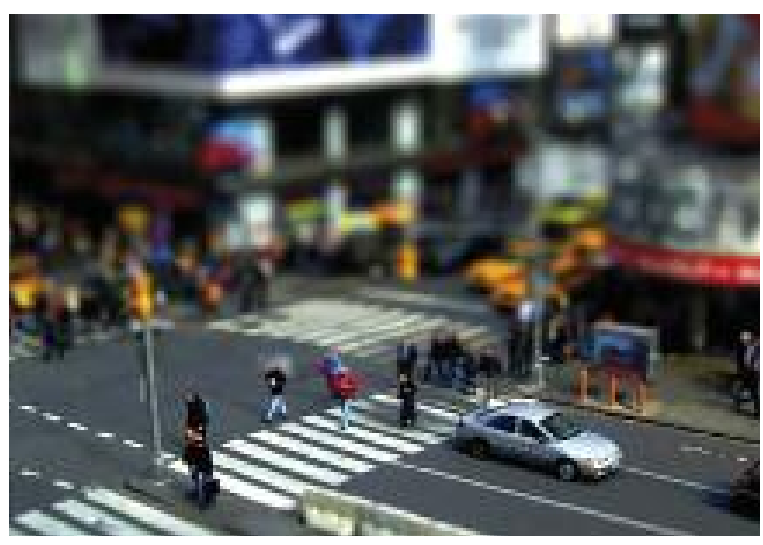

rétiniennes proches vont être disponibles et permettre la fusion sensorielle. C'est seulement si le système oculomoteur est optiquement et géométriquement bien aligné et focalisé que la perception d'une seule image tridimensionnelle du monde est réalisable. Du fait de l'écart interoculaire, différents points dans l'espace, s'ils sont vus

Figure 3. Le flou de l'arrière-plan donne un effet «maquette » à une photo standard.

traitement numérique d'image donnera un intéressant effet «maquette» à des photos standard (figure 3 ).

Absent du tableau ou de la photographie, le mouvement, ou plus précisément la parallaxe de mouvement, est un indice monoculaire fort pour la perception de profondeur. Lors d'un voyage en train, on pourra, selon son désir, fixer un point dans le paysage, et discriminer immédiatement ce qui est plus proche que ce point (semblant se déplacer à l'inverse du mouvement du train) et ce qui est plus lointain (semblant se déplacer dans le même sens). Une personne se déplaçant dans la rue, même dépourvue de vision binoculaire, pourra se repérer ou éviter les obstacles grâce à la parallaxe de mouvement et au flot optique résultant sur sa rétine.

\section{La vision binoculaire}

Pour les personnes qui en bénéficient pleinement, la vision binoculaire est un indice fort pour la perception de la profondeur. La vision binoculaire exploite les légères différences entre les points de vue de chacun des deux yeux, les disparités. Le résultat est une fusion sensorielle qui permet de ne percevoir qu'un seul percept : une seule image du monde dotée d'une information de profondeur. Cette fusion sensorielle ne peut s'effectuer que s'il y a eu au préalable fusion motrice, c'est-àdire si le système oculomoteur s'est placé dans une posture permettant à la fusion sensorielle d'opérer. Grâce à la convergence, à l'accommodation et également à l'ajustement de la pupille, deux images par les deux yeux, le seront selon deux directions différentes définissant pour chacun une disparité angulaire. Selon la posture des yeux (définie par le point de convergence, ou d'attention), ces disparités angulaires se manifesteront par des disparités rétiniennes (décalages locaux de la projection de l'espace observé sur les deux images rétiniennes). Ensuite, si l'amplitude des disparités rétiniennes correspond aux capacités de fusion de l'observateur, les deux informations issues de chacun des deux yeux pourront être fusionnées pour obtenir un percept tridimensionnel unique, par un processus de triangulation intégrant à la fois des informations oculomotrices et des informations visuelles (figure 4). Si les disparités rétiniennes sont trop importantes, par exemple pour les avant-plans ou les arrière-plans d'une scène naturelle, la fusion sensorielle n'aura pas lieu. II en résultera une double vision, ou diplopie, tout à fait commune et naturelle. Souvent, une des deux vues est annulée par un processus de suppression propre au système visuel, qui privilégiera la vue d'un œil, ou bien la vue la plus nette ou contrastée.

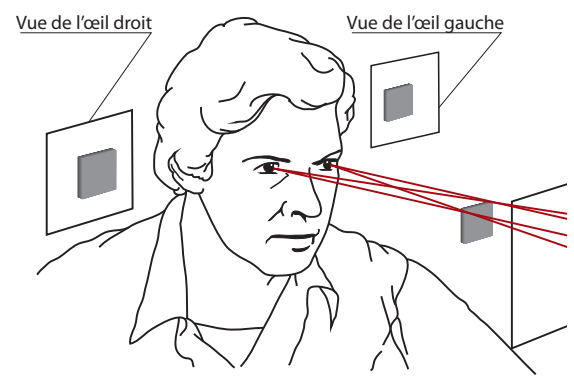

Figure 4. Les informations issues de chacun des deux yeux peuvent être fusionnées pour obtenir un percept tridimensionnel unique.

\section{TUNICS}

Lasers Accordables

TUNICS T100S-HP/CL

NOUVEAU

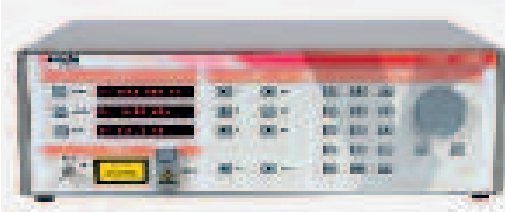

- Plage $\lambda$ : $1500-1630 \mathrm{~nm}$

- Puissance $\geq+10 \mathrm{dBm}$

- SSSER > $90 \mathrm{~dB}$

- Précision : \pm 30 pm

- Balayage sans saut de mode

\section{Autres modèles disponibles}

\section{TUNICS T100R}

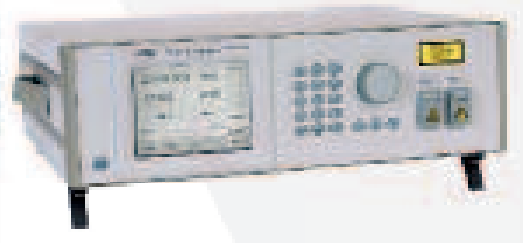

- Plage $\lambda$ : 1490 - $1650 \mathrm{~nm}$

- Précision : \pm 5 pm

\section{Technologie T100}

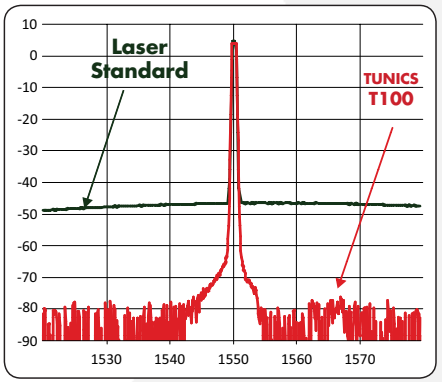

alliant haute-puissance et ultra-faible SSE

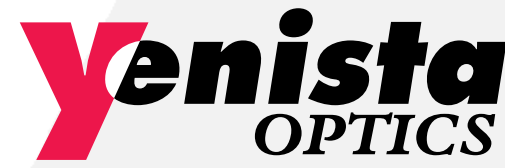

$$
\begin{gathered}
\text { Tél. : +33 (0)2964837 } 16 \\
\text { sales-emea@yenista.com } \\
\text { www.yenista.com }
\end{gathered}
$$


Dans certaines situations, par exemple lorsque deux filtres colorés différents sont placés devant les yeux ou plus généralement lorsque les deux yeux observent des images très différentes, la suppression laisse place à une rivalité binoculaire, où la vue de chaque œil peut être perçue en alternance dans une sorte de compétition.

\section{Vers une modélisation complète de la perception de la profondeur}

La perception de la profondeur est donc un processus complexe, combinant de nombreux indices oculomoteurs, binoculaires ou monoculaires. Ces indices ne doivent pas entrer en conflit afin que la vision de la scène présentée reste confortable. Ces conflits se produisent peu pour la vision naturelle où seule la rivalité binoculaire, peu fréquente, est parfois gênante. Par contre des conflits ont beaucoup plus de chance de se produire lors de la présentation d'images stéréoscopiques ou autostéréoscopiques. En effet, les images présentées ne restituent pas entièrement une scène tridimensionnelle, mais seulement une projection selon quelques directions. De plus, les images présentées correspondent à une modélisation qui reste simple de la perception humaine. L'expérience naturelle de la perception de la profondeur va au-delà de ces modèles et doit être étudiée plus avant pour améliorer encore l'expérience des spectateurs. De nouveaux écrans, de nouveaux modèles de la perception visuelle, et des contenus mieux contrôlés permettront peu à peu d'augmenter la satisfaction du public, jusqu'à ce qu'une holographie dynamique idéale permette enfin de représenter fidèlement formes et mouvements, pour la meilleure satisfaction de nos sens.

\section{Cinéma numérique}

\section{L'impulsion de la DCI}

Le cinéma numérique est né en 2005 de I'impulsion de la Digital Cinema Initiative (DCl) et de la contribution technique de nouveaux industriels du cinéma. La DCI est un regroupement des six principaux studios de cinéma, Disney, Fox, Para- mount, Sony Pictures, Universal et Warner Bros, qui ont couplé leurs efforts à partir de 2002 pour définir le cadre technique du cinéma numérique. Fruit de ces efforts, la spécification $\mathrm{DCl}$ est publiée et mise à jour depuis 2005 (voir www.dcimovies.com). Cette spécification décrit toutes les fonctions et les performances requises pour réaliser une projection numérique conforme aux exigences de qualité et de sécurité des studios : formats des données (images, sons, sous-titres, données annexes), synchronisation, multiplexage et compression, cryptage et protection des données, et enfin projection.

Ainsi, la spécification $\mathrm{DCl}$ ne décrit pas directement le système de projection dans ses détails techniques. Elle décrit les formats de données et les performances attendues. Elle a souhaité rester ouverte aux évolutions technologiques, par exemple en spécifiant une représentation de la couleur indépendante de la réalisation, basée sur un modèle standard de la perception humaine des couleurs : CIE 1931. C'est aux industriels de trouver les meilleures technologies permettant d'atteindre les performances de référence de la $\mathrm{DCl}$.

Si le système de projection en tant que tel n'est pas décrit en détails, il lui est demandé de garantir trois aspects principaux :

- la résolution et la fréquence image : $4096 \times 2160$ à $24 \mathrm{~Hz}$ (appelé 4K) ou $2048 \times 1080$ à $24 \mathrm{~Hz}(2 \mathrm{~K})$ ou $48 \mathrm{~Hz}$;

- la luminosité : niveau de luminance sur l'écran d'un blanc de référence et son uniformité ;

- la colorimétrie : définition de la chromaticité des canaux primaires rouge, vert et bleu, et du blanc, et fonction de transfert électro-optique (fonction gamma).

Ainsi, au cours de sa définition et depuis sa première publication, cette spécification du cinéma numérique a été prise en charge par des industriels qui ont conçu et développé les systèmes de cinéma que nous connaissons aujourd'hui dans nos salles. Ces nouveaux entrants de l'industrie du cinéma ont complètement transformé le monde, auparavant mécanique, des projecteurs à pellicule. Parmi eux, Texas Instruments a joué un rôle majeur en définissant le moteur optique autour de sa technologie de traitement numé- rique de la lumière DLP (digital light processing). Trois fabricants de projecteurs, Christie (Canada), Barco (Belgique) et NEC (Japon) ont intégré cette technologie dans leurs projecteurs et ont été appuyés par des fabricants de serveurs numériques (Dolby, XDC, Doremi). Pour la 3D, $d$ 'autres acteurs sont intervenus à partir de 2007, dont nous reparlerons : RealD, XPand, E3S, ou encore Dolby.

\section{Structure d'un projecteur DLP et fonctionnement 2D}

La technologie DLP de Texas Instruments est fondée sur le DMD, un composant clé inventé en 1987 par Larry J. Hornbeck. Le DMD (digital micromirror device) est un microsystème électromécanique (MEMS) composé de plusieurs millions de micro-miroirs pilotés (figure 5). Ce composant est constitué d'une mémoire CMOS sur laquelle est implantée une grille de miroirs sur pivots, un miroir pour chaque pixel. Le pas de cette grille est de l'ordre de $16 \mu \mathrm{m}$ pour le cinéma $2 \mathrm{~K}$ et chaque micro-miroir, piloté par un champ électrostatique, peut prendre deux positions mécaniques à $\pm 12^{\circ}$ de la normale du composant. Placé au sein d'un système optique, chaque micro-miroir peut donc renvoyer un faisceau incident soit vers la pupille de sortie, soit vers un piège à lumière, créant ainsi un pixel allumé ou un pixel éteint. Les miroirs étant minuscules, la commutation entre leurs deux positions peut être réalisée à plusieurs milliers de hertz. Ces deux caractéristiques, état d'illumination binaire et commutation rapide, permettent de réaliser une modulation du niveau de lumière de chaque pixel par modulation de la durée des impulsions lumineuses (PWM - pulse width modulation). La gradation des niveaux de lumière peut atteindre 12 bits, ce qui permet de répondre aux exigences de la $\mathrm{DCl}$.

Dans un système de cinéma numérique, la chaîne optique est composée d'un système d'illumination, du cœur de projection ou moteur optique et de l'objectif de projection. Pour des raisons pratiques et pour ses caractéristiques colorimétriques, la source lumineuse est la même que celle des projecteurs classiques. Il s'agit d'une lampe xénon, usuellement de 2000 à 6000 watts, dont l'arc très localisé et très 
lumineux est placé au foyer objet d'un réflecteur ellipsoïdal. Le faisceau à ce stade étant très peu uniforme, l'élément optique suivant est un barreau intégrateur, dont la section est aux proportions de la zone à illuminer sur l'écran. La face d'entrée de ce barreau est située à proximité du foyer image du réflecteur et les réflexions internes totales sur les parois latérales permettent d'ajuster l'ouverture et d'homogénéiser le faisceau pour satisfaire aux spécifications d'uniformité. La face de sortie du barreau est ensuite imagée sur les plans image par un système de renvoi et de relais, l'incidence moyenne du faisceau sur les composants DMD étant ajustée à $24^{\circ}$, comme vu plus

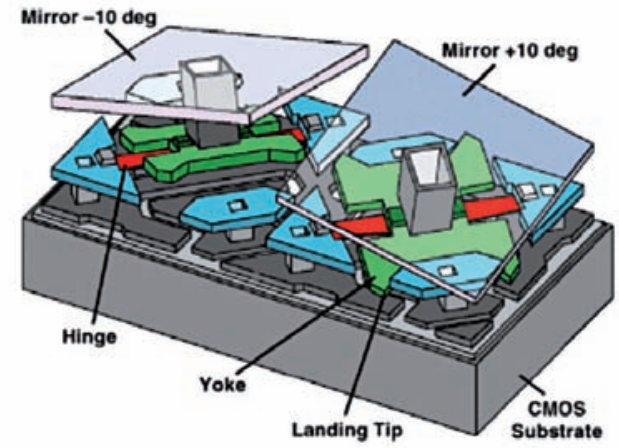

haut. Les imageurs du cœur optique sont au nombre de trois, un pour chacune des trois primaires, rouge, verte et bleue, du projecteur. La séparation spectrale est réalisée par des filtres dichroïques subdivisant les différentes voies couleur au sein d'un arrangement de prismes séparateurs.

L'objectif de projection image ensuite les DMDs sur l'écran pour des écrans jusqu'à 20 mètres de base, voire au-delà. De nombreux types d'objectifs existent donc pour répondre à des configurations diverses. Ils assurent bien entendu des fonctions de mise au point et de grandissement, mais également de correction de géométrie (trapèze) dans les deux directions, par translation parallèle au plan image, le projecteur n'étant pas forcément localisé sur la normale au centre de l'écran.

Le format $2 \mathrm{~K} / 24 \mathrm{~Hz}$ est à nos jours le format le plus utilisé comme format d'entrée pour la 2D. Le serveur fournit donc 24 images par secondes au projecteur, avec une résolution de $2048 \times 1080$ pixels, de très haute qualité car compressées en intra-image (pas de prédiction temporelle) au standard JPEG-2000 avec des paramètres de compression très stricts.

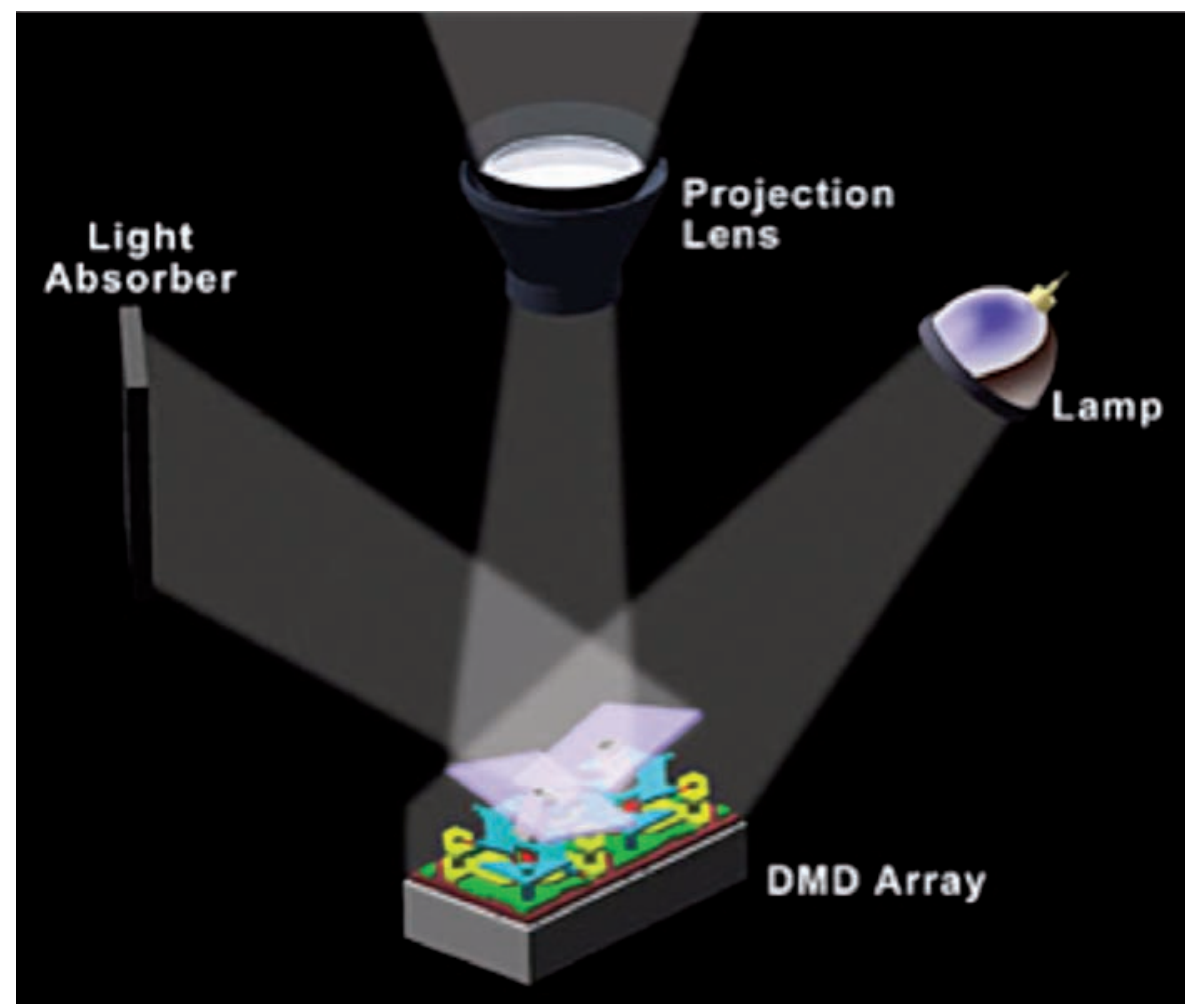

Figure 5. Schéma de principe d'un composant DMD.
Optique adaptative en mieroseopie

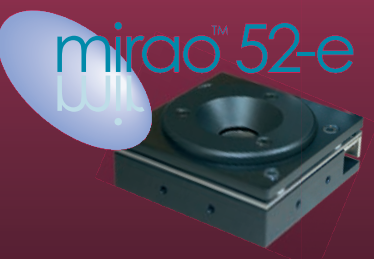

Le miroir déformable mirao 52-e, avec ou sans analyseur de surface d'onde HASO, avec nos logiciels GASAO OU GENAO, offre la meilleure capacité de correction et permet d'améliorer la résolution de limagge de manière spectaculaire.

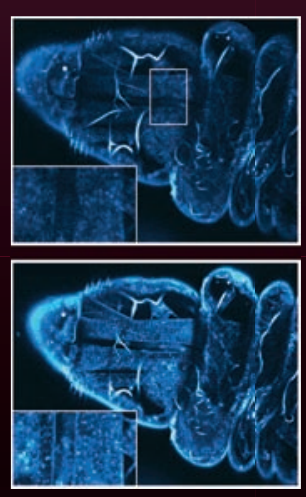

En haut, imagge d'une larve de drosophile en microscopie THG non-corrigée. En bas, image du

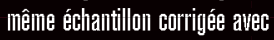
l'optique adlaptative. Images de E. Beaurepaire, D. Débarre et N. Olvier, Ecole Polytechnique, LOB

Venez nous voir en octobre sur le stanti K37 à OPTO dü 23 â 25 octobre 2012 Appelez le +33 (0)1 64861560 oul visitez :

imagine-optic.com

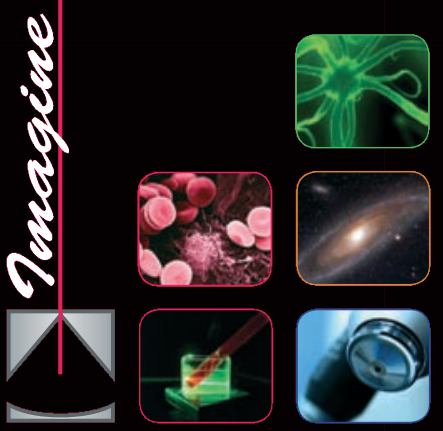

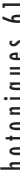


Grace à ces divers éléments, les projecteurs numériques de cinéma sont donc capables d'afficher des images de haute qualité, en correspondance avec les exigences de la $\mathrm{DCl}$, à la fois en termes de résolution, de luminosité et d'uniformité, de colorimétrie et de fréquence temporelle.

Avant d'aborder la 3D, il est à noter que, grâce à l'affichage DMD, la fréquence image de sortie peut être augmentée en répétant plusieurs fois la même image (passant à 72 ou $96 \mathrm{~Hz}$ pour la 2D, pour limiter le papillotement des scènes lumineuses).

\section{Fonctionnement 3D}

La 3D numérique au cinéma est née officiellement lors de la parution d'un document de la Digital Cinema Initiative (DCl) du 11 juillet 2007. Le Stereoscopic Digital Cinema Addendum spécifie en une demipage comment un contenu stéréoscopique doit être structuré et transmis à un projecteur de cinéma numérique. II définit, entre autres, que les trames gauche et droite doivent être transmises alterna tivement, à une cadence de 48 images par seconde, la première étant l'image gauche. Bien sûr, cet addendum ne se suffit pas à lui seul car il vient s'adosser à la très détaillée spécification $\mathrm{DCl}$ décrite brièvement au paragraphe précédent.

Pour la 3D, c'est le mode $2 \mathrm{~K} / 48 \mathrm{~Hz}$ qui est utilisé, où deux trains de données numériques sont transmis depuis le serveur jusqu'au projecteur, I'un pour la vue gauche, l'autre pour la vue droite, chacun à 24 images par seconde. Toujours afin de réduire le papillotement, le projecteur réalise un affichage en triple flash, où l'image gauche (G) est affichée trois fois, alternant avec l'image droite (D), également affichée trois fois, dans un schéma G-D-G-D-G-D-, le tout en $1 / 24^{\text {ème }}$ de seconde. Ceci donne une fréquence d'affichage image de $144 \mathrm{~Hz}$. Le double flash est également utilisé, correspondant à une fréquence de $96 \mathrm{~Hz}$.
Le mode d'affichage $2 \mathrm{~K} / 48 \mathrm{~Hz}$, alimenté par deux trains de données numériques (deux flux vidéo), permet donc de disposer d'images stéréoscopiques sur l'écran du cinéma, gauche et droite alternant à une fréquence totale de 144 images par seconde (mode triple flash).

Ceci est la première étape du cinéma numérique 3D. Cependant ces images gauche et droite sont pour l'instant superposées sur le même écran, à haute cadence, et ne peuvent être séparées à l'œil nu. Dans cette situation, sans lunettes, l'observateur verra les scènes stéréoscopiques brouillées, avec des contours doubles, et surtout n'aura aucune impression de profondeur. C'est là qu'interviennent les dispositifs à base de lunettes qui vont séparer les vues gauches des vues droites afin de les présenter convenablement à chacun des deux yeux. Pour cela il existe trois technologies principales déployées dans les cinémas, qui s'appuient sur des principes physiques différents:

- les lunettes actives exploitant la séparation temporelle des vues (XPAND et ES3) ; - les lunettes passives à polarisation (RealD) ;

- les lunettes passives à séparation chromatique (Dolby 3D).

La répartition de ces systèmes est très variable dans le monde, les États-Unis se partageant principalement entre XPAND et RealD alors que des pays comme le Royaume-Uni, la Russie ou la Chine voient un bon déploiement du système Dolby 3D. En France, les grands réseaux sont majoritairement équipés en ReaID alors que

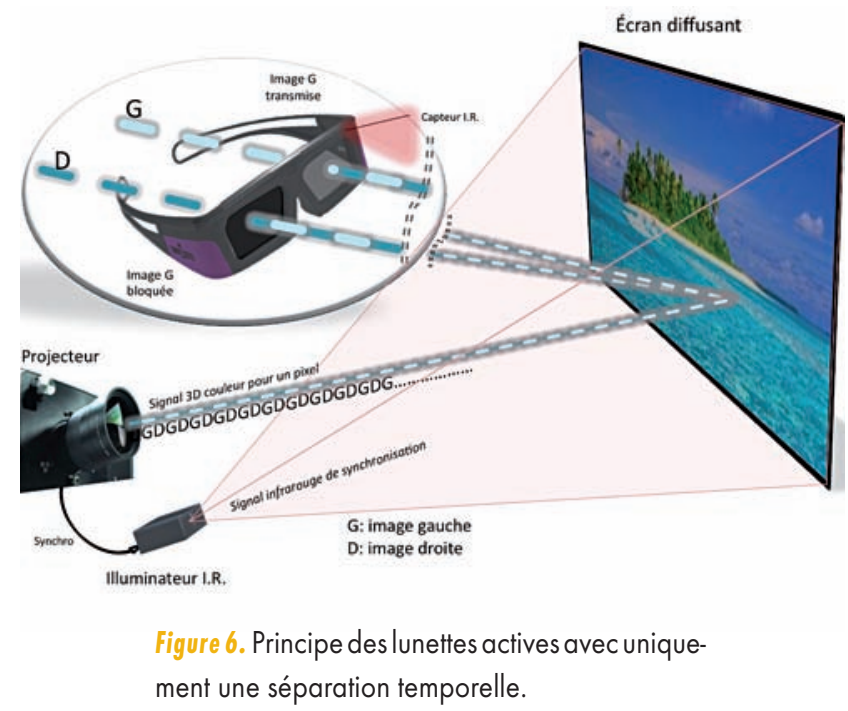

des cinémas plus exigeants en termes de qualité privilégient E3S.

\section{Lunettes actives}

Les lunettes dites actives sont les premières à avoir été mises en œuvre, en particulier par la société américaine XPAND, qui en partenariat avec Christie a équipé de nombreux cinémas dès la publication de la spécification $\mathrm{DCl}$. Ces lunettes exploitent la séparation temporelle des images gauche/droite issues des projecteurs. Chaque verre, gauche ou droite, est en fait un obturateur à cristal liquide monopixel (LCS pour liquid crystal shutter) synchronisé avec l'affichage des images gauches ou droites sur l'écran (figure 6). Ainsi, grâce à un illuminateur infrarouge connecté au projecteur, un signal de synchronisation est émis vers l'écran, qui le rediffuse vers les spectateurs. Le système de contrôle des lunettes, composé d'un capteur et d'une électronique de commande, va piloter les obturateurs à une fréquence de $72 \mathrm{~Hz}$ pour l'affichage en mode triple flash. En fonctionnement optimal, I'œil gauche ne verra que les images impaires et l'œil droit ne verra que les images paires au sens de l'addendum stéréoscopique $\mathrm{DCl}$.

Fournissant un résultat correct dans l'ensemble, cette technologie a cependant quelques limitations techniques et commerciales. Du point de vue techno logique, trois aspects ont été reprochés aux premiers systèmes mis sur le marché : une très faible luminosité, une altération des couleurs, une diaphotie entre vues (image fantôme de la voie qui devrait être bloquée), ces deux derniers défauts dépendant de la position dans la salle. De nouvelles technologies de cristaux liquides, plus rapides et isotropes, ont fortement atténué ces défauts dans les produits récents. Par exemple, Télécom Bretagne a proposé de remplacer les cellules TN (twisted nematic) par des cellules nématiques minces qui fournissent une meilleure réponse chromatique et dont les 
fronts de transition sont plus raides. Ces produits constituent l'offre E3S. Commercialement les lunettes actives présentent le désavantage d'être chères (quelques dizaines d'euros par unité) et relativement fragiles. Pour les exploitants de salles cela représente un soin particulier lors des séances 3D, du personnel et un coût supplémentaire. C'est pour cette raison qu'ont été développées des solutions à lunettes passives.

\section{Lunettes passives}

Les dispositifs à lunettes passives ont été développés comme alternative au procédé initial, afin de réduire la complexité des lunettes et de leur gestion. En effet les composants optoélectroniques des lunettes actives les rendent onéreuses. S'y ajoutent le besoin de changer ou recharger les batteries et surtout la gestion des lunettes par le personnel des cinémas. Ces contraintes sont allégées par les procédés à lu nettes passives.

Dans les systèmes DLP, les procédés passifs conservent et exploitent le séquencement des images alternant voie gauche et voie droite. Chaque voie étant projetée à un instant différent, des modifications peuvent être apportées au signal lumineux au niveau du projecteur, sur l'ensemble du faisceau lumineux, donc pour une image entière. Ces modifications, alternées, permettront ensuite aux lunettes de séparer la vue gauche de la vue droite. La lumière est estampillée ou codée par une propriété optique particulière (via sa polarisation, ou via son spectre), en fonction de l'œil destinataire, gauche ou droite. La paire de lunettes de chaque spectateur agira alors comme décodeur, transmettant le signal lumineux adapté à chaque œil (c'est-à-dire la vue gauche pour l'œil gauche, et la vue droite pour l'œil droit), et rejetant le signal

lumineux non désiré (soit la vue droite pour l'œil gauche, et la vue gauche pour l'œil droit).

\section{Lunettes passives à polarisation}

La modulation de la polarisation pour chaque alternance, gauche ou droite, du flux d'images permet d'utiliser des lunettes très peu chères (quelques dizaines de centimes d'euros), et les lunet tes sont donc facilement proposées à la vente, ou réutilisées par les spectateurs (figure 7). La société américaine RealD a développé une technologie de modulation de la polarisation appelée ZScreen, qui est placée en avant des projecteurs numériques, faisant basculer une polarisation linéaire sur deux états croisés. Ceci est réalisé à $144 \mathrm{~Hz}$, au rythme du triple flash, et en synchronisme avec l'alternance gauche/droite des images projetées. Ce dispositif est muni de lames à retard (lame quart d'onde) permettant de transformer la polarisation linéaire initiale en polarisation circulaire compatible avec les lunettes. Cette polarisation est circulaire afin d'être moins sensible à l'orientation de la tête des spectateurs.

Ce type de projection néces-

Figure 7. Lunettes passives à séparation temporelle et polarisation. site qu'un écran métallique (dit

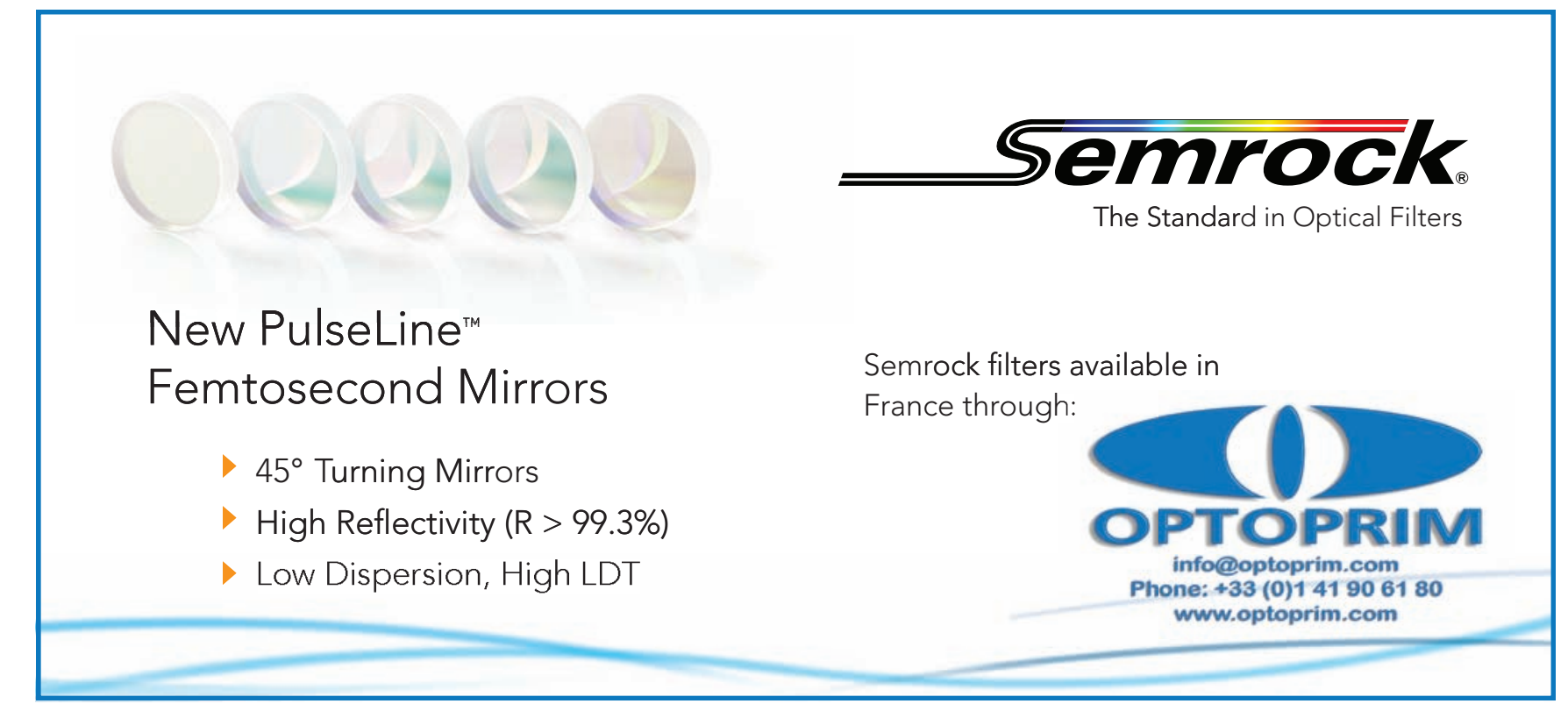


argentique) soit installé dans la salle afin de conserver la polarisation. Ce type d'écran a le désavantage d'être directif et privilégie donc les places centrales par rapport aux places latérales dans la salle. De plus, cette technologie restitue un niveau notable d'images fantômes pour lequel RealD a dû développer une technique de traitement de compensation agissant sur les images d'entrée (compensation croisée entre image gauche et image droite pour les régions à fort contraste en disparité). Avec ce procédé, la faible luminosité, une certaine influence chromatique et une diaphotie persistent.

La polarisation circulaire, et donc les lunettes 3D passives, sont utilisées également par deux systèmes de projection n'utilisant pas la technologie DLP. Tout d'abord, Sony a développé un projecteur LCOS (cristal liquide sur silicium) à double résolution, 4K (4096 $\times 2160$ pixels), dans lequel deux images, gauche et droite, peuvent être agencées l'une au-dessus de l'autre sur l'afficheur LCOS pour être projetées simultanément sur l'écran par un objectif spécifique. L'alternance temporelle n'est plus nécessaire et chaque image gauche ou droite peut-être polarisée indépendamment, avant projection, ce qui permet ensuite une séparation par des lunettes passives à polarisation. Le procédé Technicolor 3D-film est tout à fait similaire, plaçant deux images gauche et droite l'une au-dessus de l'autre sur la pellicule et utilisant un objectif double corps pour les afficher sur la même surface d'écran, mais avec des polarisations opposées, donc séparables avec les lunettes passives.

\section{Lunettes passives à séparation chromatique}

Simultanément au développement de la 3D passive basée sur la polarisation, Infitec, une entreprise de technologie allemande, a inventé une technique de séparation chromatique pouvant bénéficier de la plate-forme DLP. Devenue Dolby 3D, cette technologie se base sur une séparation des voies gauche et droite dans le

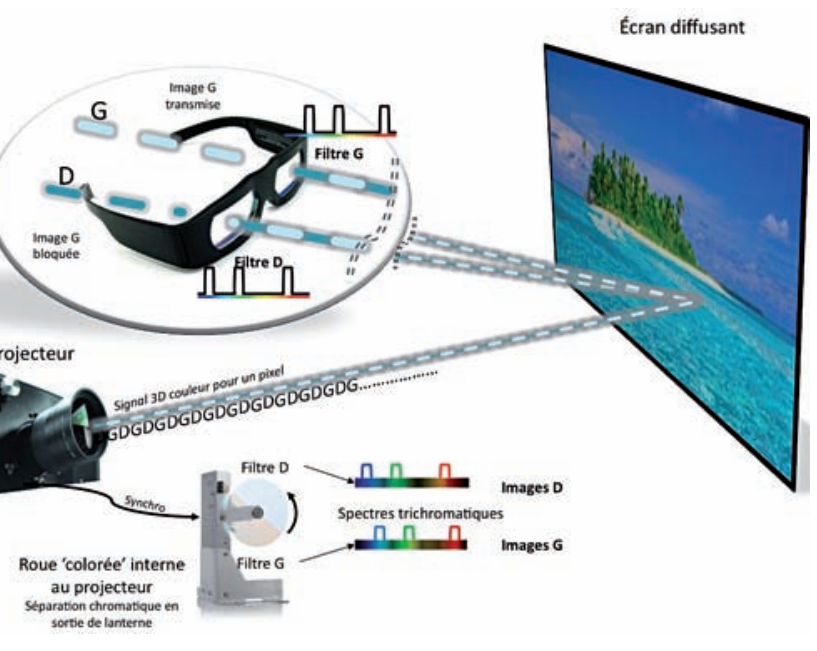

les lunettes. Malgré cela, Dolby $3 \mathrm{D}$ a réussi à s'implanter fortement dans de nombreux pays dont la Chine et la Russie.

\section{Les contenus 3D}

Comme nous l'avons vu, les projecteurs numériques $3 \mathrm{D}$ sont des systèmes technologiques performants permettant d'afficher des vues stéréoscopiques en conformité avec la spécification $\mathrm{DCl}$, c'est-à-dire avec un très haut niveau de qualité.

Figure 8. Lunettes passives à séparation temporelle et chromatique.

Nous avons vu également que la perception de la profondeur mettait en œuvre des mécanismes complexes, où l'expérience individuelle compte pour beaucoup et présentant une sensibilité variable à différents conflits d'indices 3D que peuvent présenter les images.

Il est donc nécessaire, lors de la création de contenus, de bien comprendre et de respecter la vision humaine dans sa complexité, au risque de créer un rejet de la part du public. C'est un travail qui est en cours, au niveau des studios et de leurs partenaires techniques, qui implique la formation des équipes de tournage à une nouvelle scénographie, à de nouvelles techniques, et la création de nouveaux outils de captation, de traitement et de contrôle.

après calibration (fournissant la même couleur perçue au sens d'un observateur standard). La rotation de la roue synchronisée à 72 tours par seconde sur la projection des images gauches et droites envoie sur l'écran un faisceau appartenant alternativement à I'un ou à l'autre des deux espaces chromatiques, qui peuvent être séparés par des filtres adaptés placés sur les lunettes.

La technologie Infitec/Dolby 3D présente des performances techniques re marquables, avec très peu d'images fantômes et sans dépendance vis-à-vis de la position dans la salle. La luminosité reste faible, et la difficulté principale reste que le coût des lunettes, qui n'est pourtant que de quelques euros, ne permet pas d'avoir le même modèle d'usage que RealD qui évite aux exploitants de gérer

Références
http://www.dlp.com/technology/how-dlp-
works/default.aspx
http://www.ti.com/lit/an/dlpa022/dlpa022.pdf
http://www.xpand.me/technology/xpand-3d
http://www.eyes3shut.com/technology
http://www.reald.com/content/cinemaPro-
ducts.aspx
http://pro.sony.com/bbsc/ssr/mkt-digitalcinema
http://www.technicolor.com/en/hi/theatrical/3d-
in-the-theatre
http://www.dolby.com/us/en/professional/tech-
nology/cinema/dolby-3d.html
http://www.technicolor.com/en/hi/3d/certifi3d
«Cinéma, la conquête de la 3D», Thierry Borel,
magazine «Pour la Science» ${ }^{\circ} 416$, juin 2012,
pp. 56-63

